\title{
Impurity Efferts on Ion-Drift-Wave Eigenmodes in a Sheared Magnetic Field
}

\author{
W.M. Tang, R.B. White, and P.N. Guzdar \\ Plasma Physics Laboratory, Princeton University \\ Princeton, New Jersey 08544
}

The stability of ion-drift-wave eigenmodes in a slab geometry with a sheared magnetic field is investigated. It is found that in contrast to the case of universal and dissipative electron dxift wave eigenmodes, unstable impurity-driven normal modes can appear if specific conditions are satisfied. In addition, the influence of impurities on unstable ion-temperature-gradient-driven drift eigenmodes is also studied. It is found that, if their density profile is inwardly peaked, the impurities can exert a strong stabilizing influence on these modes.

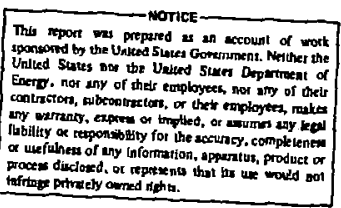




\section{INTRODUCTION}

The influence of radially nonlocal effects, such as magnetic shear, on the presence of absolutely unstable drift modes in a confined plasma is a fundamental problem that has been actively investigated in numerous papers. Most of these studies have been carried out for a slab geometry with shear and have focused on electron drift waves with long radial wavelengths. This approach leads to a differential eigenmode equation of the form

$$
\left[\partial^{2} / \partial x^{2}+Q(x, \omega)\right] \phi(x)=0
$$

with $Q(x, w)$ being the governing radial potential, whose specific form is determined by the particular instability of interest. In the case of universal (collisionless) ${ }^{l}$ and dissipative (collisional) drirt waves, 2 it has been emphasized in several recent papers that, if the complete electron dynamics driving these modes is properly included in $Q(x, w)$, then absolutely unstable forms of these instabilities cannot be present at long radial wavelengths $\left(k_{x} \rho_{i}<1\right.$ with $p_{i}$ being the ion gyroradius). In the present paper, it is demonstrated that unlike these modes, the impurity-driven ion-drift waves, ${ }^{3,4}$ previously derived in only the radially local limit, can persist as unstable normal modes in a sheared slab geometry provided certain criteria are satisfied. Additionally, the influence of impurities on unstable ion-temperature-gradient-driven drift eigenmodes $^{5-8}$ is also determined.

Before describing the radially nonlocal calculations, it is appropriate to first, review the characteriscic features of ion-drift waves driven unstable by impurity effects and by ion temperature 
gradients. As noted in the original local derivation of the impurity modes by coppi, et al., ${ }^{3}$ the basic requirement for their onset is that in addition to the electron and principal ion (taken here to be hydrogen) populations, there must be an impurity population with a density gradient which is opposicely directed from those of the main plasma components. A representative example of this type of configuration is one where the electron and hydrogen components monotonically decrease away from the center of the plasma $(x=0)$, and the impurity component is peaked toward the outside. For such equilibrium density profiles, the diamagnetic drift frequency, $\omega_{* j} \equiv\left(k_{Y} c T / Z e B_{O} L_{n}\right)_{j}$ with $L_{n} \equiv-\left(d \ln n_{o} / d r\right)^{-1}$, will be positive for the electrons and impurities $\left(\omega_{* e}, \omega_{* I}>0\right)$ and negative for the principal ion component $\left(\omega_{* i}<0\right)$. It should also be remembered here that charge neutrality must be preserved; i.e.,

$$
\sum_{j}\left(z^{2} n_{0} \omega_{\star} / T\right)_{j}=0
$$

For the ion-temperature-gradient modes, the basic requirement is simply that $n_{i} \equiv d \ln T_{i} / d$ In $n_{O_{i}}$ be sufficiently large. As pointed out by Kadomtsev and Pogutse ${ }^{5}$ (and confirmed in later more detailed studies ${ }^{7}$ ) the criterion is roughly $n_{i} \geq 1$.

In the local analysis 3,4 of impurity drift instabilities, it was found that the dominant modes of this type have characteristic parallel phase velocities below the thermal velocity of hydrogen but above that of the impurity species; i.e., $\mathbf{v}_{\mathrm{TI}}<\omega / k_{11}<\mathrm{v}_{\mathrm{Ti}}$. Hence, to a good approximation, the perturbed electron density response can be taken as adiabatic; i.e., $\left(n / n_{0}\right)_{e}=\mid e / \phi / T_{e}$. The hyarogen ions, on the other hand, now have a role analogous to 
that of the electrons for universal and dissipative drift modes. Specifically, the perturbed density response of hydrogen provides the destabilizing contribution in the form of inverse Landau damping in the collisionless regime ${ }^{3}$ and collisional dissipation in the more collisional regimes. ${ }^{4}$ As shown in Ref. 3, the local analysis indicates that the impurity drift modes typically propagate in the ion diamagnetic direction [i.e., $\operatorname{Re}(\omega) \simeq \omega_{* i}$ ] and have maximum growth rates of roughly the same magnitude as $\operatorname{Re}(\omega)\left[i . e ., \operatorname{Im}(\omega) \simeq-\omega_{\star i}\right]$. In the next section a radially nonlocal analysis of these modes as well as the $\eta_{i}$-ariven eigenmodes in the presence of impurities will be carried out. The results will be presented in Sec. III, and the conclusions and implications of these studies discussed in Sec. IV.

\section{EIGENMODE ANALYSIS}

To derive the basic eigenmode equation, standard procedures ${ }^{3}$ can be employed to calculate the full kinetic density response of the electrons and the ion components. For the regime of interest $\left(v_{T I}<\omega / k_{1}\right)$ prescribed in earlier studies, 3,4 the impurity response can also be obtained using the fluid approximation, i.e.,

$$
\frac{n_{I}}{n_{O I}}=-\frac{z_{I}|e| \phi}{T_{I}}\left(\frac{\omega_{\star I}}{\omega}+\frac{k_{\perp}^{2} \rho_{I}^{2}}{2}-\frac{k_{I}^{2} v_{I}^{2}}{2 \omega^{2}}\right) .
$$

Although the complete kinetic response of the electrons was taken into account in the calculations carried out in this paper, it was found that using the simple linearized Boltzmann response, $n_{e} / n_{o e}=|e| \phi / T_{e}, y i e l d s$ essentially the same results. For the 
ions, the perturbed density response in the collisionless limit can be expressed in the form:

$$
\begin{gathered}
\frac{n_{i}}{n_{0 i}}=-\frac{|e| \phi}{T_{i}}\left\{I+\left(1-\frac{\omega_{* i}}{\omega}\right) \zeta_{i} z\left(\zeta_{i}\right) \Gamma_{0}-\frac{w_{* i}}{\omega} n_{i} \zeta_{i}\left[\zeta_{i}+\left(\zeta_{i}^{2}-\frac{1}{2}\right] z\left(\zeta_{i}\right)\right] \Gamma_{0}\right. \\
\left.+b\left(\Gamma_{i}-\Gamma_{0}\right) z\left(\zeta_{i}\right)\right\}
\end{gathered}
$$

with $z\left(\zeta_{i}\right)$ being the plasma dispersion function, ${ }^{9} \zeta_{i} \equiv \omega / k_{1}, v_{i}$, and $\Gamma_{n} \equiv I_{n}(b) \exp (-b)$ with $I_{n}(b)$ being the modified Bessel function and $b \equiv k_{\perp}^{2} \rho_{i}^{2} / 2$.

The familiar sheared slab configuration ${ }^{1,2}$ considered has an equilibrium magnetic field of the form, $B=B_{0}\left[\hat{z}+\left(x / L_{s}\right) \hat{y}\right]$, with $I_{s}$ being the shear length and $x$ corresponding to the radial variable. Hence, for perturbations of the form, $\phi=\phi(x) \exp \left(-i \omega t+i k_{y} y\right)$, the parallel wavenumber becomes $k_{1}=k_{y} x / L_{s}$. At long radial wavelengths $\left(k_{x} \rho_{i}<1\right)$, the quasineutrality condition, $\sum_{j} z_{j} e_{j} n_{j}=0$, together with the assumption that the radial variation in equilibrium quantities such as $\omega_{* j}$ can be ignored, leads to the radial differential eigenmode equation given in Eq. (1); i.e.,

$$
\left[\partial^{2} / \partial x^{2}+Q(x, w)\right] \phi(x)=0
$$

where $x$ is now a dimensionless variable $(x \rightarrow x p s / \sqrt{2})$ and

$$
Q(x, w)=A(x, w) / B(x, w)
$$

with 


$$
\begin{aligned}
& A(x, w)=\frac{n_{o e}}{n_{o i}}-z_{I} \frac{n_{O I}}{n_{O i}} \frac{1}{\Omega} \frac{L_{n e}}{L_{n I}}+b \tau \frac{n_{O I}}{n_{O i}} \frac{M_{I}}{m_{i}}-z_{I}^{2} \frac{n_{O I}}{n_{o i}} \frac{m_{i}}{M_{I}}\left(\frac{I_{n e}}{L_{s}}\right)^{2} \frac{x^{2}}{\Omega^{2}} \\
& +\tau+\tau\left[\left(1+\frac{1}{\Omega \tau} \frac{L_{n e}}{L_{n i}}\right) \Gamma_{0}-\frac{\eta_{i}}{2 \Omega \tau} \frac{L_{n e}}{L_{n i}}\left[\Gamma_{0}-2 b\left(\Gamma_{1}-\Gamma_{0}\right)\right]\right] \zeta_{i} z\left(\zeta_{i}\right) \\
& +\frac{\eta_{i}}{\Omega} \frac{L_{n e}}{L_{n i}} \Gamma_{o} \zeta_{i}^{2}\left[1+\zeta_{i} z\left(\zeta_{i}\right)\right]
\end{aligned}
$$

$B(x, \omega)=-\left[\left(1+\frac{1}{\Omega \tau} \frac{L_{n e}}{L_{n i}}\right)\left(\Gamma_{1}-\Gamma_{0}\right)-\frac{n_{i}}{2 \Omega \tau} \frac{L_{n e}}{L_{n i}}\left[\Gamma_{0}+\Gamma_{1}+4 b\left(\Gamma_{1}-\Gamma_{0}\right)\right]\right] \zeta_{i} z\left(\zeta_{i}\right)$

$$
-\frac{\eta_{i}}{\Omega \tau} \frac{L_{n e}}{\Sigma_{n i}}\left(\Gamma_{1}-\Gamma_{0}\right) \zeta_{i}^{2}\left[I+\zeta_{i} z\left(\zeta_{i}\right)\right]-\frac{n_{o I}}{n_{o i}} \frac{M_{I}}{m_{i}}
$$

$\Omega \equiv \omega / \omega_{t e}, \tau \equiv T_{e} / T_{i}$, and $\zeta_{i} \equiv \Omega\left(L_{s} / L_{n e}\right)(\tau / 2)^{1 / 2} /|x|$. This equation determines the radial eigenfunctions and eigenvalues of both the impurity-driven eigenmodes and (by suppressing the impurity terms) the $\eta_{i}$-driven eigenmodes. 8

\section{A. Local Analysis}

The radially local dispersion relation can be easily recovered by treating $k_{11}=k_{y} \times / L_{s}$ as a simple input variable in $\mathrm{Eq}$. (6) and then setting $A(w)=\bar{u}$. To solve for eigenvalues of the impurity drift modes, it is convenient to adopt the approximations made by Coppi, et al. ${ }^{3} i . e .$, ignore the finite gyroradius and impurityion-acoustic terms and take

$$
z\left(\zeta_{i}\right) \simeq i \pi^{1 / 2} \zeta_{i}\left[1-\left(\omega / \omega_{* i}\right)\left[1-\left(\eta_{i} / 2\right)\right]\right\}
$$

with $\left|\zeta_{i}\right| \ll 1$. This leads to the result 


$$
\omega=-\frac{z_{I}^{2} I_{I}}{T_{I}} \omega_{\star I}\left\{\frac{n_{o e}}{T_{e}}+\frac{n_{o i}}{T_{i}}+i \pi^{1 / 2} \zeta_{i}\left[1-\frac{\omega}{\omega_{\star i}}\left(1-\frac{\eta i}{2}\right)\right]\right\}^{-1}
$$

Following the earlier calculation, ${ }^{3}$ first note that at marginal stability, $\omega_{0}=\omega_{*_{i}}\left[1-\left(n_{i} / 2\right)\right]$, and then substitute $\omega=\omega_{0}+\delta \omega_{r}+i \gamma$ back into $\mathrm{Eq.}$ ( (8) to obtain

$$
\delta \omega_{I}=-\omega_{* i}\left(1-\frac{n_{i}}{2}\right)-\frac{z_{I}^{2} \circ I}{T_{I}} \omega_{* I}\left(\frac{n_{o e}}{T_{e}}+\frac{n_{o i}}{T_{i}}\right)^{-1},
$$

and

$$
\gamma \approx \frac{z_{I}^{2} n_{O I}}{T_{I}} \omega_{\star I} \frac{\pi^{1 / 2} \delta \omega_{r}}{k_{11} v_{i}}\left(\frac{{ }^{n} o e}{T_{e}}+\frac{{ }^{n} o i}{T_{i}}\right)
$$

Hence, for instability, the criterion reduces to $\omega_{* I}{ }^{\omega_{r}}>0$ or

$$
\frac{\omega_{* i}}{\omega_{* I}}\left(1-\frac{n_{i}}{2}\right)+\frac{z_{I}^{2} n_{o I}}{T_{I}}\left(\frac{n_{o e}}{T_{e}}+\frac{n_{o i}}{T_{i}}\right)^{-1}>0 \text {. }
$$

Noting that the second term is generally much less than unity, and setting $n_{i}=0$, this is just the requirement ${ }^{3}$ that the impurity density gradient be oppositely directed from those of the main plasma componentsi i.e., $L_{n I} / L_{n i}<0$.

Although the simple estimates here lead to results which will prove to be in qualitative agreement with those from the nonlocal caiculations, it should be pointed out that such a procedure can also generate erroneous conclusions. In particular, if the $n_{i}$ term is retained in $\mathrm{Bg}$. (II), the inference from this type of calculation would be that positive ion temperature gradients $\left(n_{i}>0\right.$ ) should exert a stabilizing influence on the impurity modes. However, when the complete nonlocal analysis is carried out, it is found that just the opposite is true; i.e., $n_{i}>0$ is destabilizing 
while $\eta_{i}<0$ is stabilizing. This is a consequence of the fact that the $B(x, w)$ term, specified in Eq. (7), has a different $\eta_{i}$ dependence than $A(x, \omega)$. As noted earlier, this factor does not appear in the simple local calculation.

The local analysis of ion-temperature-gradient driven modes is well documented in earlier papers. 5,7 The simplest form of these instabilities (ignoring resonant ion effects) can be readily obtained from fluid equations. 5,7 Inclusion of resonant ion effects leads to an $\eta_{i}$ threshold near unity. ${ }^{7}$ As before, the local results for $n_{i}$-modes can be recovered from $\mathrm{Eq}$. (6) by ignoring the impurities, treating $k_{11}$ as an input variable, and setting $A(\omega)=0$.

\section{B. Nonlocal Analysis}

Before describing the procedures used to carry out the radially nonlocal calculations, it is appropriate to first comment on the nature of the governing potential, $V(x, w) \equiv-Q(x, w)$ in Eq. (1). In contrast to the familiar "anti-well" form of $v$ for the electron drift waves, ${ }^{1}$ the real part of the potential for the ion drift waves can have the shape of a bounded well. Hence, instead of appearing as propagating eigenmodes characteristic of electron drift waves, these ion waves can take the form of dominantly nonpropagating normal modes.

The above point can be illustrated by considering the simplest form of the $n_{i}$-driven instability ${ }^{7}$ which requires $n_{i} \gg 1$ and has $\left|r / \omega_{r}\right| \gg 1$ with $\omega_{r}{ }^{\alpha \omega_{* i}}$. The potential for this case reduces to the usual Weber equation form, 6 


$$
V(x, \omega)=-Q(x, \omega)=A(\omega)-B(\omega) x^{2}
$$

with

$$
A(\omega)=\frac{\tau(\Omega-1)}{\Omega \tau+1+\eta_{i}}, B(\omega)=\left(\frac{L_{n}}{L_{s}} \frac{1}{\Omega}\right)^{2},
$$

$\tau \equiv T_{e} / T_{i}$, and $\Omega \equiv \omega / \omega_{* e}$. Notice here that for $\left|\gamma / \omega_{r}\right|>>1$, the potential, $V$, is dominantly real and has the shape of a well; i.e., $\operatorname{Re}(V)=\operatorname{Re}(A) x^{2}$ with $\operatorname{Re}(B)<0$. In addition, since $\Omega_{r}<0$ and $n_{i}>1$ impiy that Re(A) $<0$, the governing potential is a bounded well. Hence, the solutions to the differential equation will accordingly take on the character of dominantly nonpropagating normal modes. Although the actual form of $Q(x, w)$, as given in Eq.'s $(5-7)$, is far more complicated than Eq. (12), the basic bounded-well nature of the potential is found to persist.

In carrying out the detailed radially nonlocal analysis, solutions were obtained by the WKBJ or phase integral method ${ }^{10}$ and also by a standard shooting code procedure. ${ }^{11}$ The WKBJ solution to Eq. (1) has the familiar form

$$
\left.\phi_{ \pm}=Q^{-1 / 4} \exp \{ \pm i\}^{z} d z Q^{1 / 2}\right\}
$$

with $z=x+i y$ being the spatial varıable in the complex plane, $Q$ being defined in Eq.'s $(5-7)$, and the boundary conditions determining the proper combination of $\phi_{+}$and $\phi_{-}$. As shown in Ref. 10, the corresponding eigenvalue equation is

$$
\int_{z_{1}}^{z_{2}} d z[Q(z, w)]^{1 / 2}=\left(n+\frac{1}{2}\right) \pi
$$


with $n$ being the radial mode number and $z_{1}, z_{2}$ being the turning points determined by $Q(z, \omega)=0$.

From Eq. (13) it is clear that the solution, $\phi$, is oscillating along the so-called "anti-stokes lines" which are defined by the requirement that the integral in the exponential be real. Accordingly, a local anti-Stokes line (at $z=z_{0}$ ) can be represented as an infinitesimal path emanating from $z_{0}$ along which $Q^{1 / 2} \mathrm{dz}$ is real. Provided $Q\left(z_{0}, w\right)$ is finite and well behaved, such a line is simply determined by setting $d z_{c}$ equal to a real number times $\pm\left[Q\left(z_{0}\right)\right]^{-1 / 2}$. Hence, from each local point $z_{0}$ in the complex plane, there will issue two oppositely directed lines. In the vicinity of a turning point $\left\{z=z_{T}\right.$ with $\left.Q\left(z_{T}\right)=0\right\}, Q(z) \simeq\left(z-z_{T}\right) Q^{\prime}\left(z_{T}\right)$. Since this implies the requirement that $\left(z-z_{T}\right)^{3 / 2}$ be real near $z_{T}$, there will now be three lines emanating from this local point. As described in detail by white, ${ }^{12}$ the local anti-stokes lines can be used to form the global stokes diagram which in turn describes the global properties of the WKBJ solutions. The actual WKBJ calculation implemented can be briefly summarized as follows. First, an initial guess for $\omega$ is taken from the local analysis and used to generate the global stokes diagram. If the diagram indicates two good turning points and a structure consistent with the boundary condition requirement that the solutions be exponentially decaying at large $x$ (subdominant regions), then the eigenvalue condition, given in Eq. (14), is used to iteratively search for the proper $\omega$. A typical picture of a Stokes diagram corresponding to an unstable normal mode is given in Fig. 1 .

The shooting code procedure used to complement and check the WKBJ analysis involves the application of a well-known finite 
difference scheme known as the Numerov algorithm. 11 Hers an initial guess for $w$ is used in the UKRJ form for $\phi$ given in Eq. (13) with the appropriate sign chosen to correspond to exponentially decaying solutions. Equation (1) is then integrated from large $x$ (where the WKBJ form is a good approximation) to the origin $(x=0)$. The required boundary condition at the origin provides the constraint determining the eigenvalue. Specifically, $d \phi /\left.d x\right|_{x=0}=0$ for even modes and $\phi(0)=0$ for odd modes. The new value of $\omega$ predicted here is then used as the initial guess, and the entire procedure is repeated until the scheme converges.

\section{III, RESULTS}

In this section the eigenmode analysis described in the preceding section is applied to the impurity-driven and $\eta_{i}$-driven ion arift instabilities. Results presented here deal with the influence of magnetic shear, typical wave number spectra, collisional effects, and the combined effects of impurities and ion temperature gradients.

\section{A. Impurity Drift Eigenmodes}

In general the radial localization of the impurity-driven eigenmodes is governed by the dynamics of the principal ion component (taken to be hydrogen). Specifically, it is the ion $z$-function response in Eq.'s (6) and (7) which determine the relevant turning points for these normal modes. Typical eigenfunctions are plotted on Fig. 2 for radial mode numbers $n=0$ and $n=2$. In these cases the chosen input parameters are $\tau=T_{e} / T_{i}=1, b=k_{y}^{2} \rho_{i}^{2} / 2=0.125$, $n_{i}=0, L_{n e} / L_{s}=0.1, z_{I} n_{o I} / n_{o e}=0.1, z_{I}=8$ (oxygen), and $I_{n e} / L_{n I}=-8$ 
(to correspond to outwardly peaked impurity density profile). The qualitative dependence of these instabilities on wave number (b), magnetic shear strength $\left(L_{n e} / L_{s}\right)$, and ion temperature gradient ( $n_{i}$ ) can be conveniently illustrated by varying the physical parameter of interest while keeping the rest fixed to those values just specified for Fig. 2 .

A typical wave number $\left(\mathrm{k}_{\mathrm{y}}\right)$ spectrum for impurity eigenmodes is plotted on Fig. 3. It is clear from this graph that over a wide range of wavelengths where these instabilities are strongest (i.e., for $b<11$, they appear to be quite insensitive to changes in the wave number. Hence, for the rest of the parameter variation studies carried out here, $\mathrm{b}=0.125$ is chosen as a representative case.

The influence of magnetic shear on the impurity eigenmodes is illustrated on Fig. 4. Here $s$ seen that these modes remain absolutely unstable over a wide range of shear strength $\left(L_{s} / L_{n e}\right)$. For the more realistic values of $L_{s} / L_{\text {ne }}$ (i.e., $L_{s} / L_{\text {ne }}$ lying between 10 and 50 ) the $n=2$ radial eigenmode is found to have the largest growth rate. As shown on Fig. 4, the actual sensitivity of this mode to changes in $\mathrm{L}_{s} / L_{\text {ne }}$ (between 10 and 50 ) is not pronounced. It should also be noted that for the very weak shear regime $\left(L_{s} / L_{n}>50\right)$, the higher radial eigenmodes $(n>2)$ will be the dominant instabilities. In addition to the shear dependence of the impurity eigenmodes, Fig. 4 also illustrates the good agreement between the results from the WKBJ and shooting code calculations.

As mentioned earlier in this paper, if the ion temperature gradient $\left(n_{i} \neq 0\right)$ is taken into account in the analysis, it is found that for $\eta_{i}<0$ the influence is stabilizing, while for: $\eta_{i}>0$ the effect is destabilizing. This $\eta_{i}$-dependence of the impurity 
eigenmodes is piotted on Fig. 5. Here it is seen that the destabilizing trend actually sets in for $n_{i}>I$. of course, even in the absence of impurities, the condition $\eta_{i}>1$ is sufficient to drive ion drift waves unstable.?

In order to study the behavior of impurity eigenmodes in a more collisional regime, a simple number-conserving form of the Krook collision operator is employed. 4 For $n_{i}=0$ and $b \ll 1$, the terms in the governing potential specified in Eq.'s (6) and (7) become:

$$
\begin{aligned}
A(x, w)=\frac{n_{o e}}{n_{o i}}-z_{I} \frac{n_{o I}}{n_{O i}} \frac{1}{\Omega} \frac{L_{n e}}{L_{n I}}+b \tau & \frac{n_{o I}}{n_{O i}} \frac{M_{I}}{m_{i}}-z_{I}^{2} \frac{n_{o I}}{n_{o i}} \frac{m_{i}}{M_{I}}\left(\frac{L_{e}}{L_{s}}\right)^{2} \frac{x^{2}}{\Omega^{2}} \\
& +\tau+\tau(1-b) E\left(w, v_{i}\right),
\end{aligned}
$$

$B(x, w)=E\left(w, v_{i}\right)-\frac{n_{o I}}{n_{o i}} \frac{M_{I}}{m_{i}}$,

with

$E\left(\omega, v_{i}\right) \equiv \frac{\left(1+\frac{1}{\Omega \tau} \frac{L_{n e}}{L_{n i}}\right) \frac{a \Omega}{|x|} z\left(\frac{a\left(\Omega+i \bar{\nu}_{i}{ }^{\prime}\right.}{|x|}\right)}{1+\frac{i_{i} \bar{v}_{i}}{|x|} z\left(\frac{a\left(\Omega+i \bar{\nu}_{i}\right)}{|x|^{\prime}}\right)}$,

$\bar{v}_{i} \equiv v_{i i} / w_{* e}$ (accounting for hydrogen-hydrogen collisions) and $a \equiv\left(L_{s} / I_{n}\right)(\tau / 2)^{I / 2}$. As in the previous radially local calculation, 4 electron-hydrogen and impurity-hydrogen collisions are taken to be negligible. Typical results of the radially nonlocal analysis is shown on Fig. 6. The qualitative trend indicated here is that the collisional effects do not significantly modify the collisionless results. For example, a very large collision frequency $\left(v_{i i}>5_{u}{ }_{\star}\right)$ 
is required to reduce the growth rate by about a factor of two.

\section{B. Ion Temperature Gradient Drift Eigenmodes}

In the absence of impurities, detailed numerical (shooting code) solutions to Eq.'s $(5-7)$ have been obtained by Waltz, et al. 8 On the basis of these results, it was concluded that if $\eta_{i}>1$, then unstable normal modes can readily appear and are quite insensitive to shear stabilization. For a typiral magnetic shear strength, $L_{n e} / L_{s}=0.1$, and $\eta_{i}=2$, their calculations indicated that the largest growth rate is associated with the fundamental ( $n=0)$ radial eigenmode. In the present calculations, it is found that this dominance of the $n=0$ mode (for $L_{n e} / L_{s}=0.1$ ) persists at larger values of $\eta_{i}$. Hence, for the remainder of this section attention will be focused on results dealing with the $n=0$ eigenmodes with $I_{n e^{/ L}}=0.1$.

A typical wave number spectrum for the $\eta_{i}$-modes (with $\eta_{i}=3$ ) is illustrated on Fig. 7. Here it is seen that these instabilities are quite insensitive to changes in $k_{y}$ for $b=k_{y}^{2} \rho_{i}^{2} / 2<0.1$, and that the largest growth rates occur around $b=0.5$.

Using $b=0.5$, the sensitivity of these temperature-gradientdriven eigenmodes to the parameter $n_{i}$ was studied. As shown on Fig. 8, the destabilizing influence of increasing this variable is approximately linear up to $\eta_{i} \simeq 3$. However, at higher values of $n_{i}$, the growth rates of these modes become almost independent of this parameter. 
On the basis of the preceding results, it seems appropriate to choose the $n=0$ radial eigenmode with $I_{n e} / L_{s}=0.1, b=0.5$, and $\eta_{i}=3$ as a representative case to study the influence of impurities. As noted earlier in this paper, the presence of impurities with outwardly peaked density profiles will in general enhance the destabilization of ion drift waves. However, if the impurities are inwardly peaked, their stabilizing influence can be quite dramatic. This favorable trend is most easily seen in the analytic treatment of these modes ignoring resonant ion effects. As shown, for example, by Coppi, et al. ${ }^{6}$ the resultant weber equation for the $n_{i}$ eigenmodes leads to the eigenvalue condition,

$$
-i \frac{L_{n e}}{L_{s}} \frac{(2 n+1)}{\Omega}=\frac{\Omega \tau-\tau}{I+n_{i}+\Omega T}
$$

with $\eta_{i} \gg 1$ required for self-consistent unstable solutions. If impurities are now included, the eigenvalue equation becomes

$$
-i \frac{L_{n e}}{L_{s}} \frac{(2 n+1)}{\Omega}=\frac{\delta \tau \frac{n_{o e}}{n_{o i}}-\tau\left(\frac{L_{n e}}{I_{n i}}+z_{I} \frac{n_{o I}}{n_{o e}} \frac{L_{n e}}{L_{n I}}\right)}{\left(I+\eta_{i}\right) \frac{L_{n e}}{L_{n i}}+\Omega \tau} .
$$

with

$$
\frac{L_{n e}}{L_{n i}}=\frac{n_{o e}}{n_{o i}}\left(1-z_{I} \frac{n_{o I}}{n_{o e}} \frac{L_{n e}}{L_{n I}}\right)
$$

as a consequence of charge neutrality. Obviously, in the absence of impurities, $L_{n e}=L_{n i}$ and $n_{o e}=n_{o i}$, so that $E q$. (18) just reduces to $\mathrm{Eg}$. (17). However, if the impurities are present 
and inwardly peaked $\left(L_{n e} / L_{n I}>0\right)$, then $I_{n e} / L_{n i}<1$. Since $I_{n e} / L_{n i}$ multiplies the destabilizing term, $1+n_{i}$, the effect here is clearly stabilizing. This favorable trend is also found to persist when solving the more exact eigenmode problem as specified by Eq.'s (5-7). The stabilizing influence of inwardly peaked impurities on the $n_{i}$-driven instabilities is illustrated on Fig.'s 9 and 10 . For a given impurity concentration $\left(z_{I^{n} I_{O}} / n_{o e}=0.2\right)$, the required degree of peaking for stabilization is shown on Fig. 9 to be about $L_{n e} / L_{n I} \geq 2$. on Fig. 10, the required impurity concentration for stabilization corresponding to several different inwardly peaked impurity profiles is plotted. It is seen that for the more stronqly peaked cases, the impurity concentration needed for stability is quite low. For example, with $\mathrm{L}_{n e^{/ L_{n I}}}>2$, the required $\mathrm{z}_{I} \mathrm{n}_{\mathrm{oI}} / \mathrm{n}_{\mathrm{oe}}<0.2$. This corresponds' to less than a three percent density concentration of a low-z impurity, such as oxygen.

\section{CONCLUSIONS}

A systematic analysis of long radial wavelength $\left(k_{x}{ }_{i}<1\right)$ ion drift wave eigenmodes in a sheared slab geometry has been presented in this paper. With regard to impurity-driven modes, it is found that for outwardly peaked impurity profiles, these instabilities are very unlikely to be shear stabilized. On the other hand, if the impurities are inwardly peaked, the instabilities do not appear. Moreover, for such a situation, the impurities tend to exert a strong stabilizing effect on the temperature-gradient-driven ion drift modes. In light of these results, it would be quite interesting to explore the possible existence of favorable impurity 
profiles in actual experiments. For example, Goldston's measurements ${ }^{13}$ on the ATC (adiabatic toroidal compressor) tokamak indicate an inward peaking of the effective z-profile. This at least allows for the possibility that individual types of low- 2 impurities could be inwardly peaked. In general, measurements of such individual .mpurity profiles on existing experiments should provide some very useful information.

ACKNOWLEDGMENT

This work was supported by the United States Department of Energy Contract No. EY-76-C-02-3073. 


\section{REFERENCES}

1. D.W. Ross and S.M. Mahajan, Phys. Rev. Lett. 40,324 (1978); K.T. Tsang, P.J. Catto, J.C. Whitson, and J. Smith, Phys. Rev. Lett. 40,327 (1978); T. Antonsen, Phys. Rev. Lett. 4I, 33 (1978); L. Chen, P.N. Guzdar, R.B. White, P.K. Kaw, and C. Oberman, Phys. Rev. Lett. 41,649 (1978).

2. P.N. Guzdar, L. Chen, P.K. Kaw, and C. Oberman, Phys. Rev. Lett. 40,1566 (1978).

3. B. Coppi, H.P. Furth, M.N. Rosenbluth, and R.Z. Sagdeev, Phys. Rev. Lett. 17, 377 (1966).

4. S.K. Wong, Phys. Fluids 18, 391 (1975).

5. I.I. Rudakov and R.z. Sagdeev, Soviet Piıs., Doklady 7,417 (1961).

6. B. Coppi, M.N. Rosenbluth, and R.Z. Sagdeev, Phys. Fluids 10, 582 (1967).

7. B.B. Kadomtsev and O.P. Pogutse, in Reviews of Plasma Physics, edited by M.A. Leontovich (Consultants Bureau, New York, 1970), Vol. 5, p. 303 .

8. R.E. Waltz, W. Pfeiffer, and R.R. Dominquez, General Atomic Co. Rept. GA-A15147 (Dec., 1978).

9. B.D. Fried and S.D. Conte, The Plasma Dispersion Function, (Acäß̊mic, New York, 1961).

10. J. Heading, An Introduction to Phase Integral Methods, (Wiley, New York, 1962). 
11. R.W. Hamning, Numerical Methods for Scientists and Engineers, (McGraw-Hill, New York, 1962), p. 215.

12. R.B. White, J. Comput. Phys. 31, No. 3 (1979).

13. R.J. Goldston, Phys. Fluids 21, 2346 (1978). 


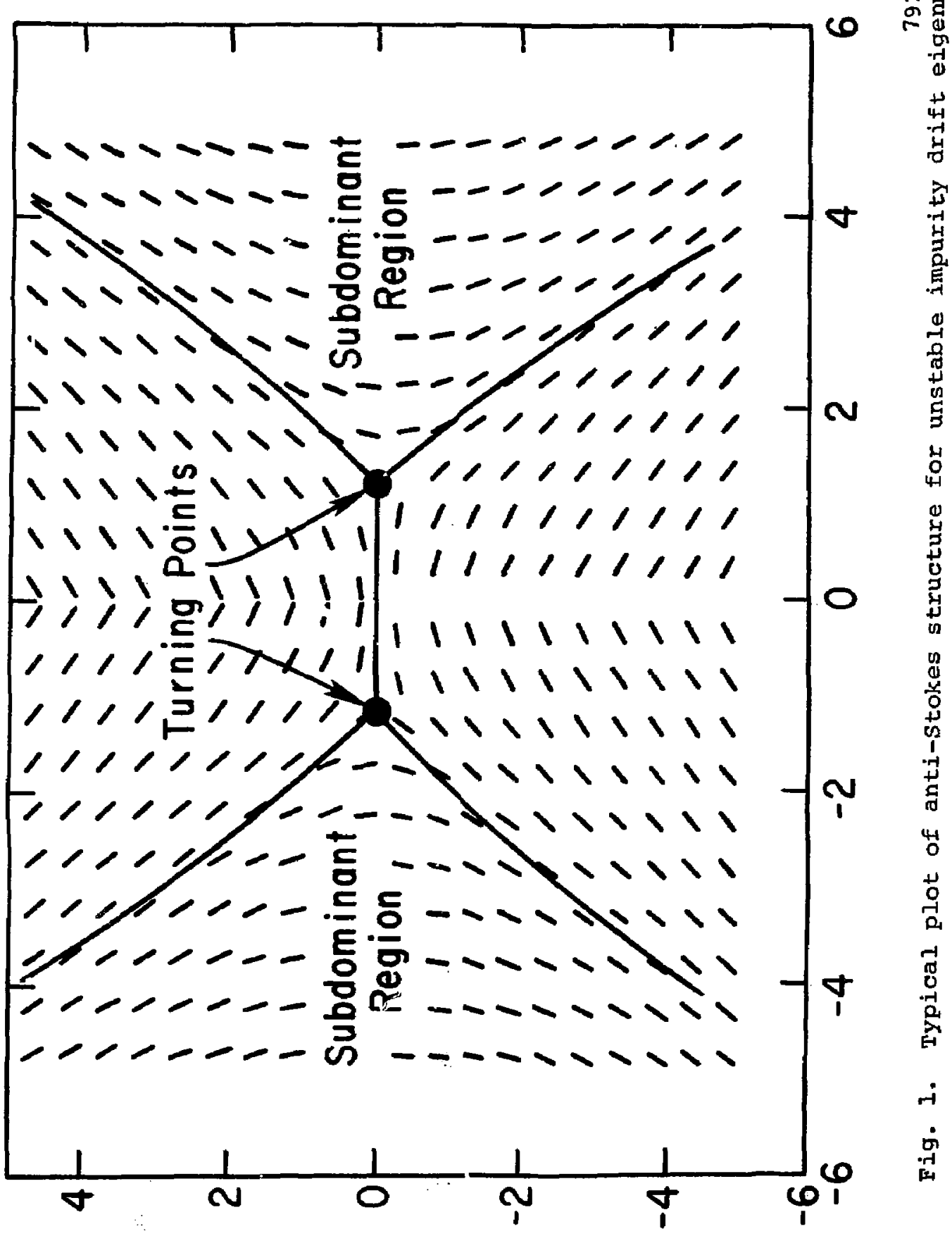



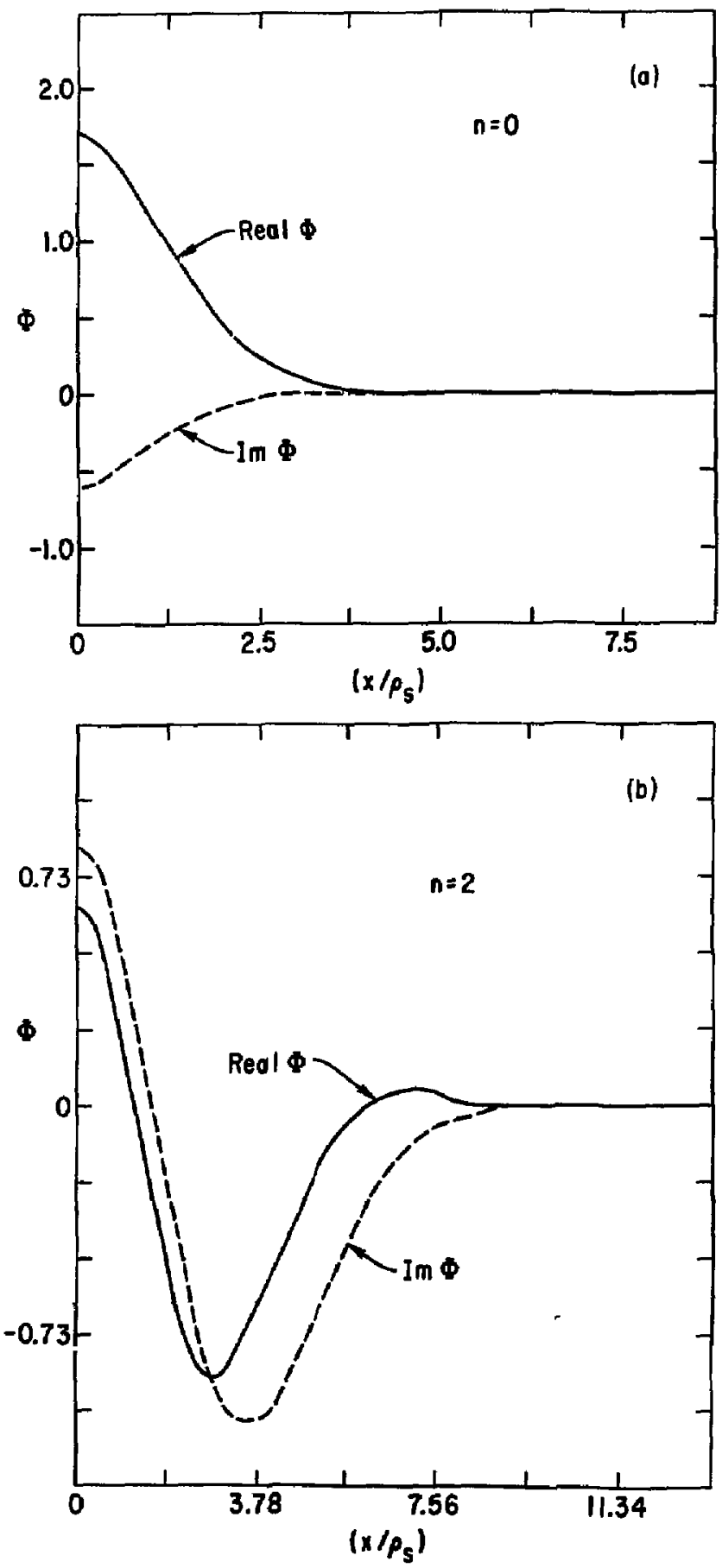

Fig. 2. Typical olots of $n=0$ and $n=272129$ eigenfunctions for impurity drift instability. 


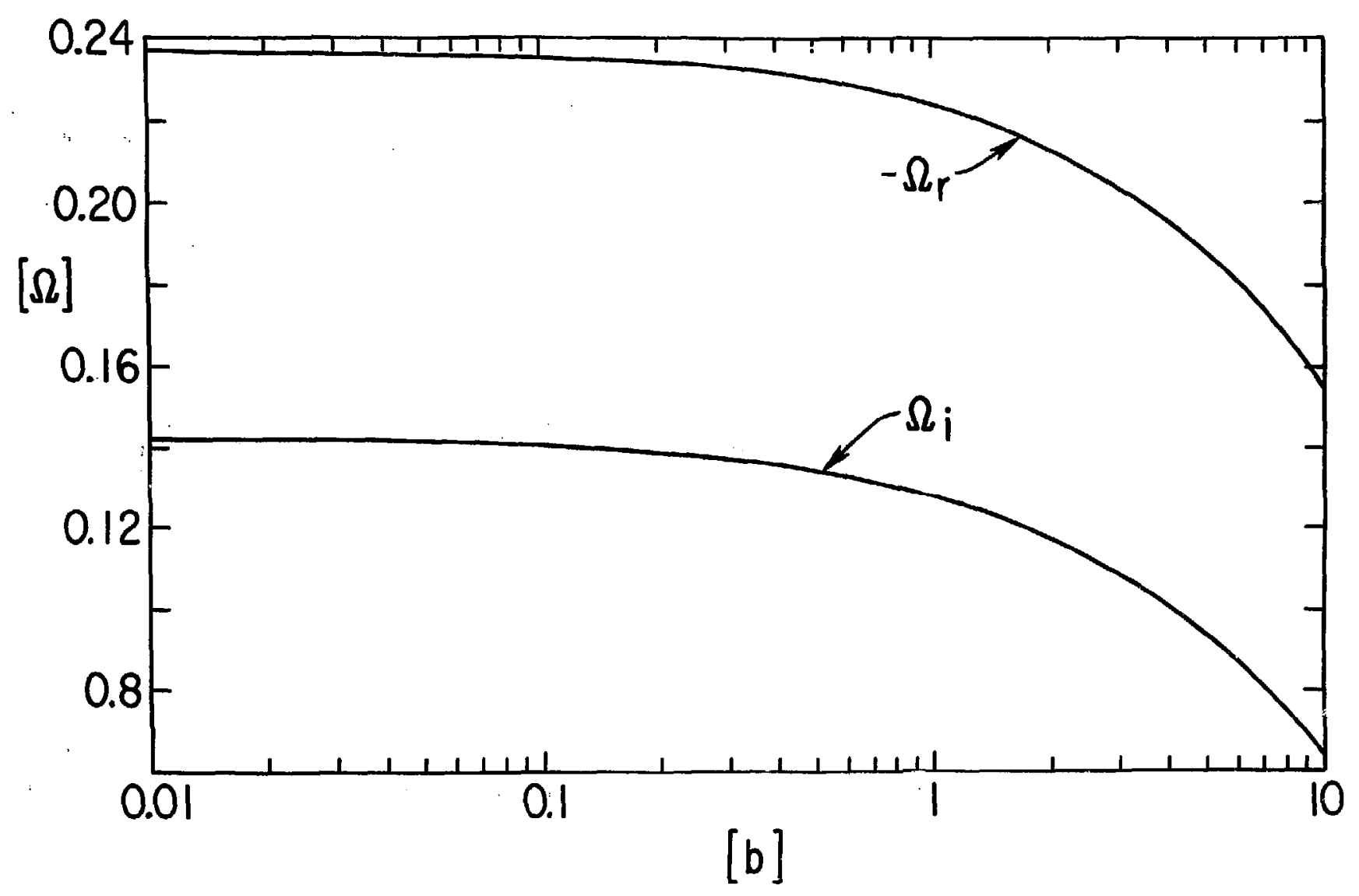

792126 Fig. 3. Typical wave number $\left(b \equiv k_{y}^{2} p_{i}^{2} / 2\right)$ spectrum for impurity drift instability. 


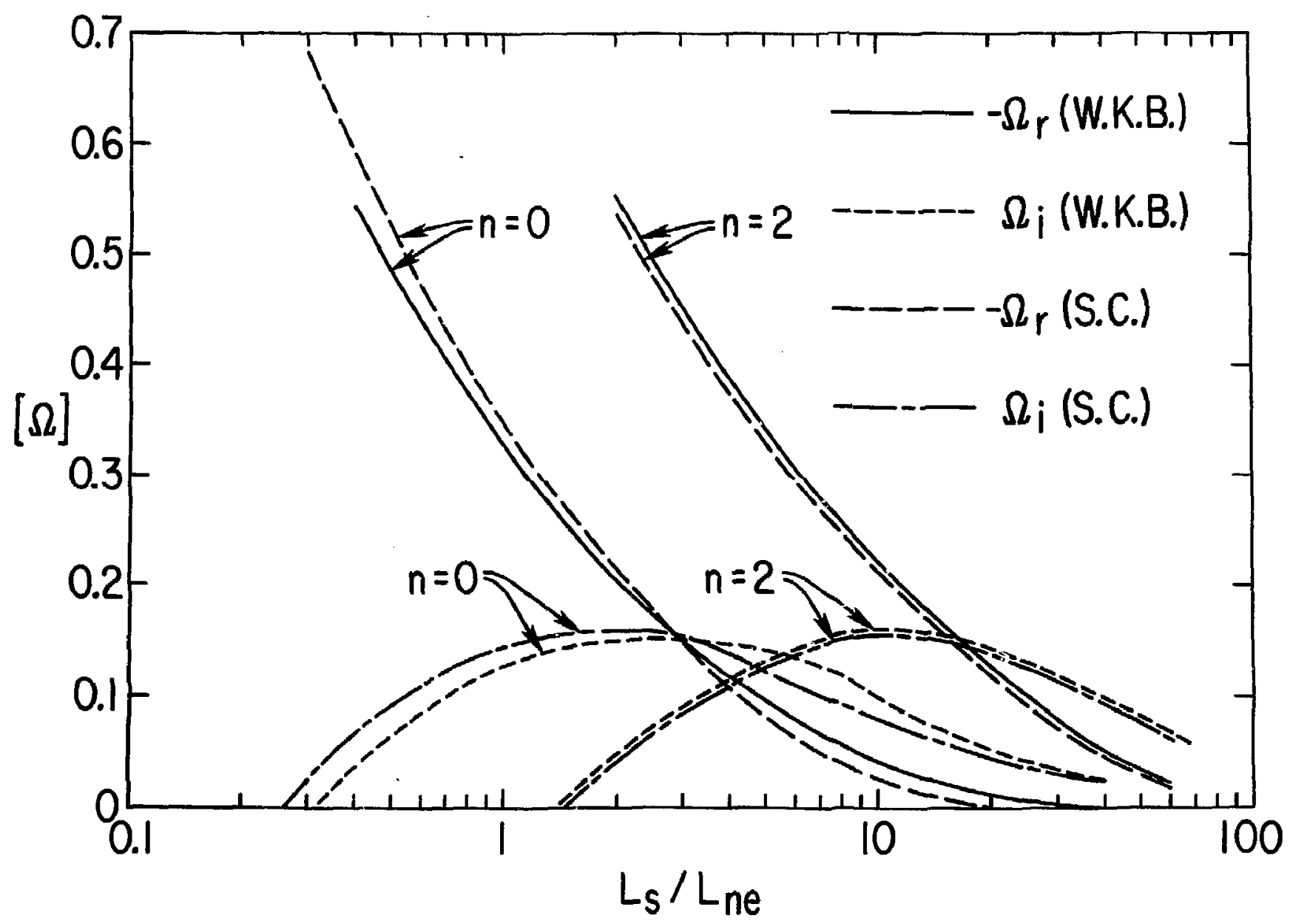

792130

Fig. 4. Results from WKBJ and shooting code calculations showing typical shear dependence of the $n=0$ and $n=2$ impurity drift radial eigenmodes. 


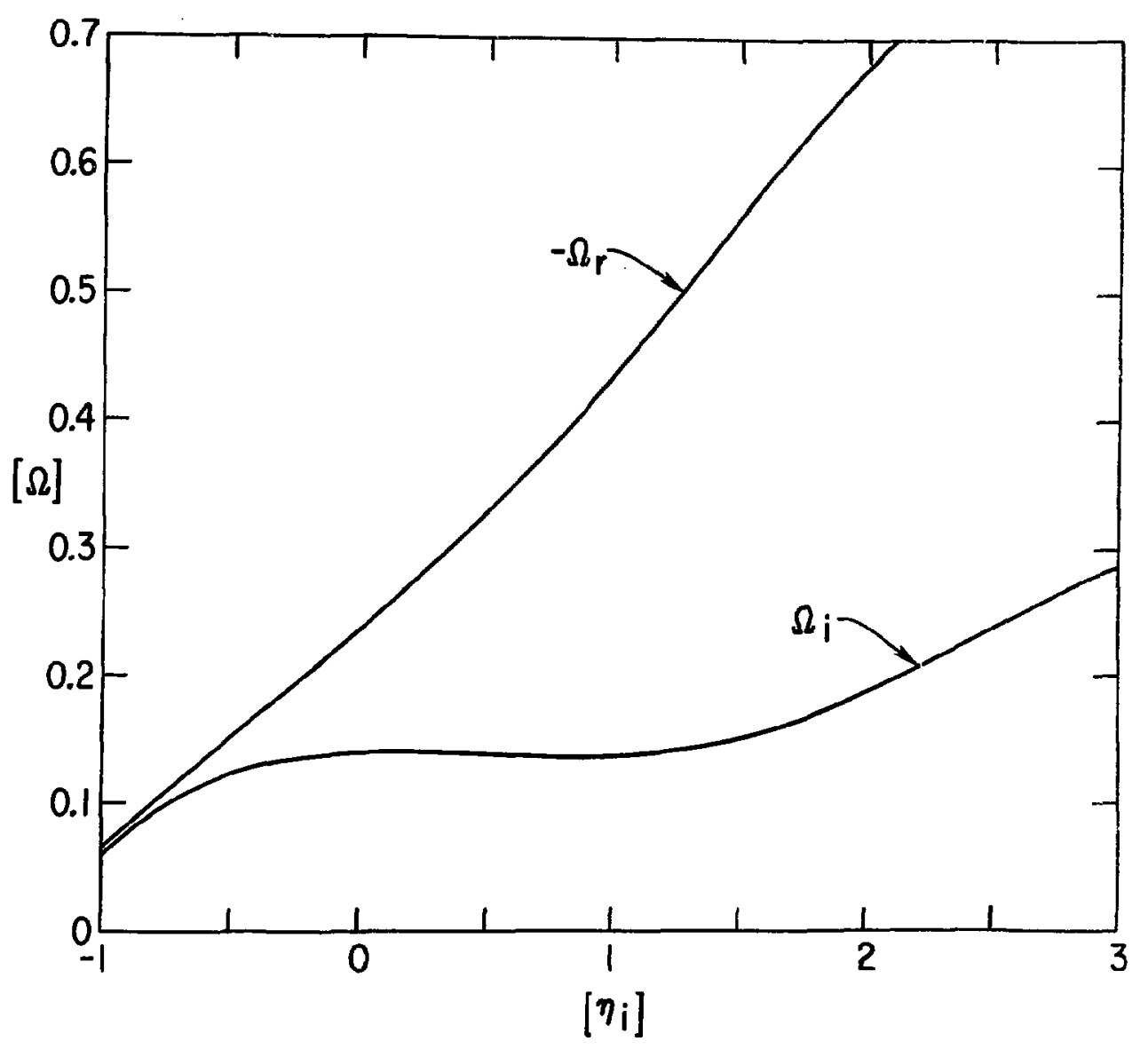

792123

Fig. 5. Typical ion temperature gradient dependence of impurity drift instability. 


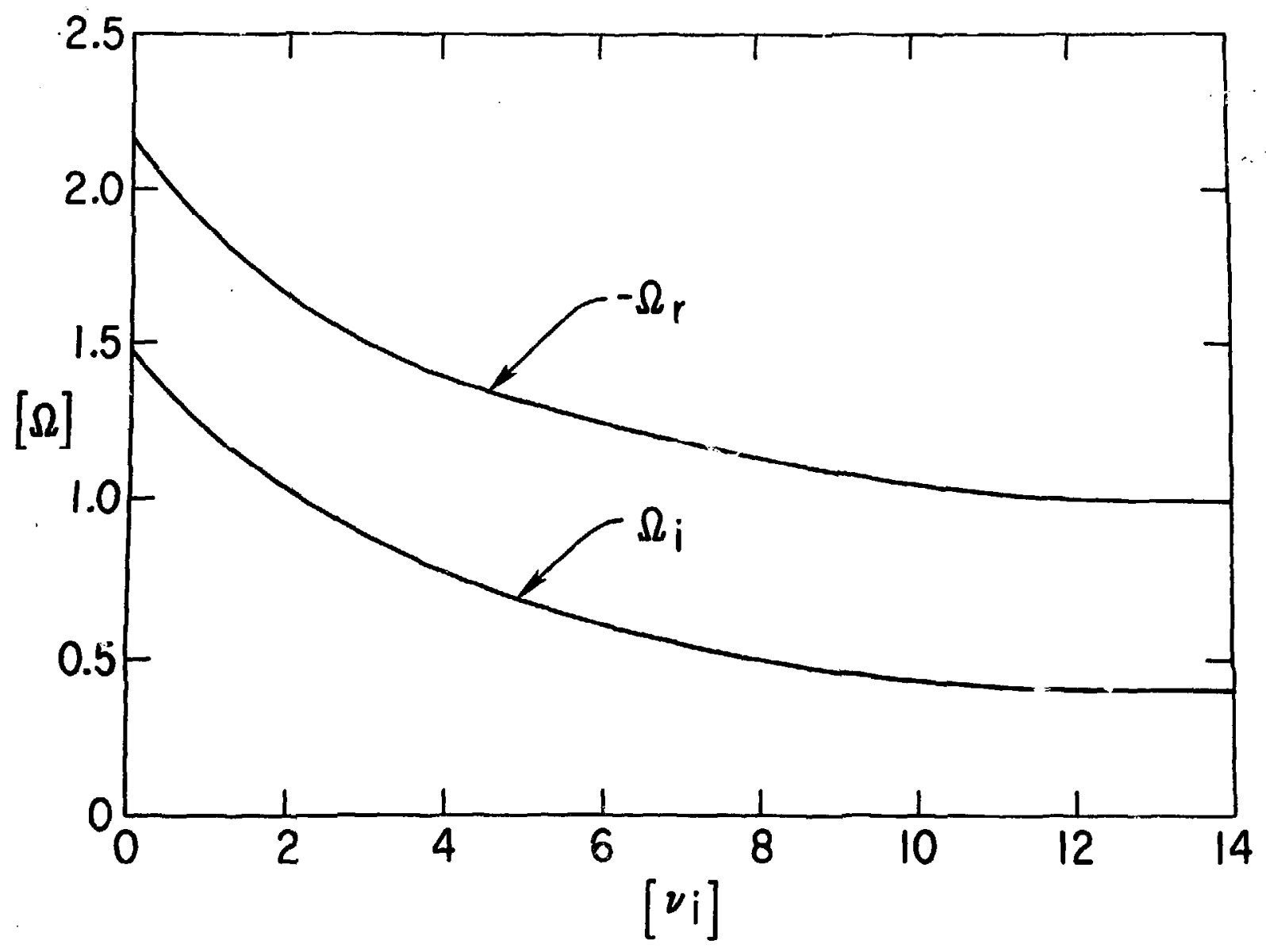

Fig. 6. Typical dependence of impurity drtift instability on ion-ion (hydrogen) collisions. 


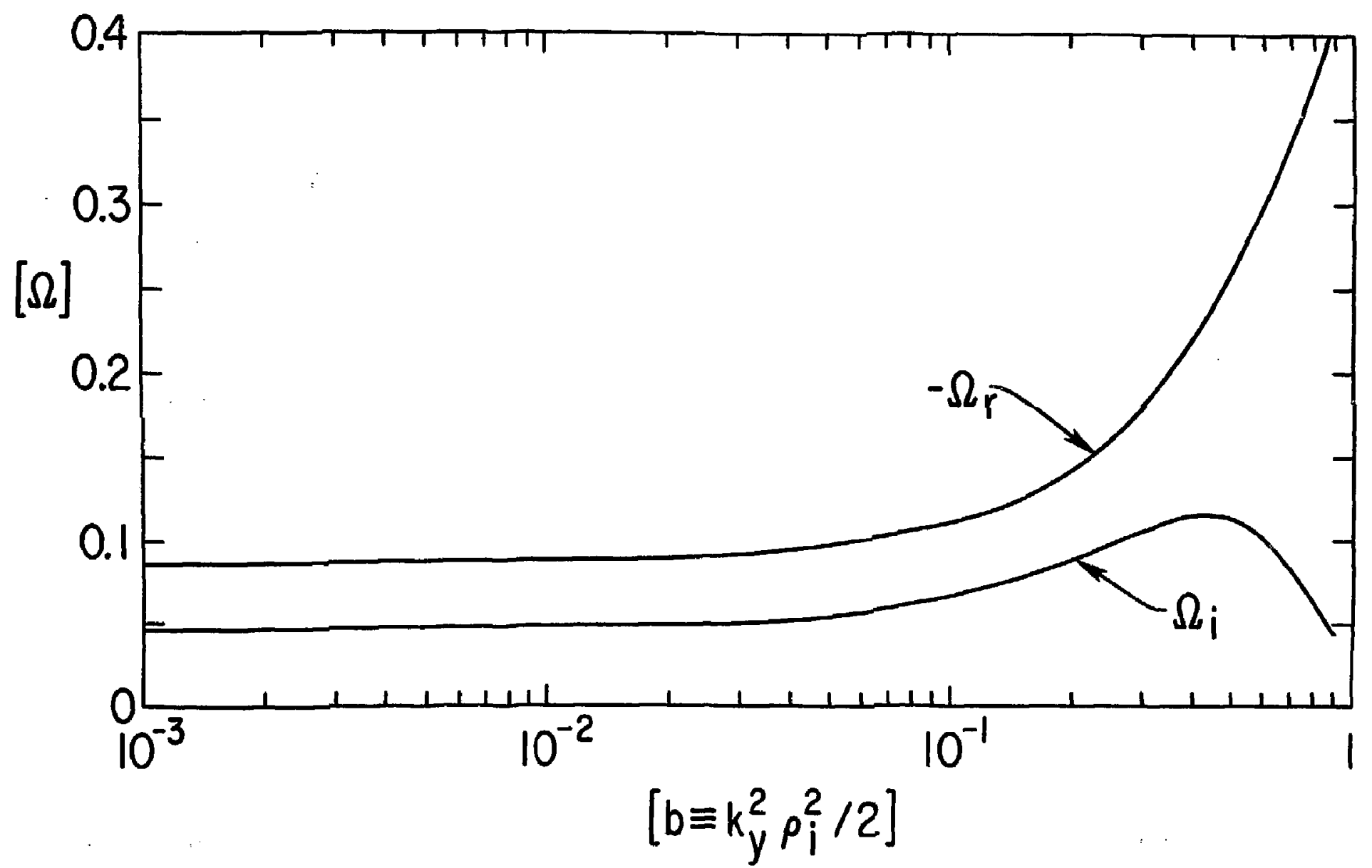

792127

Fig. 7. Wave number (b) spectrum for ion temperature gradient instability with $n_{i}=3, L_{n} / L_{s}=0.1$, and radial mode number $(n)=0$. 


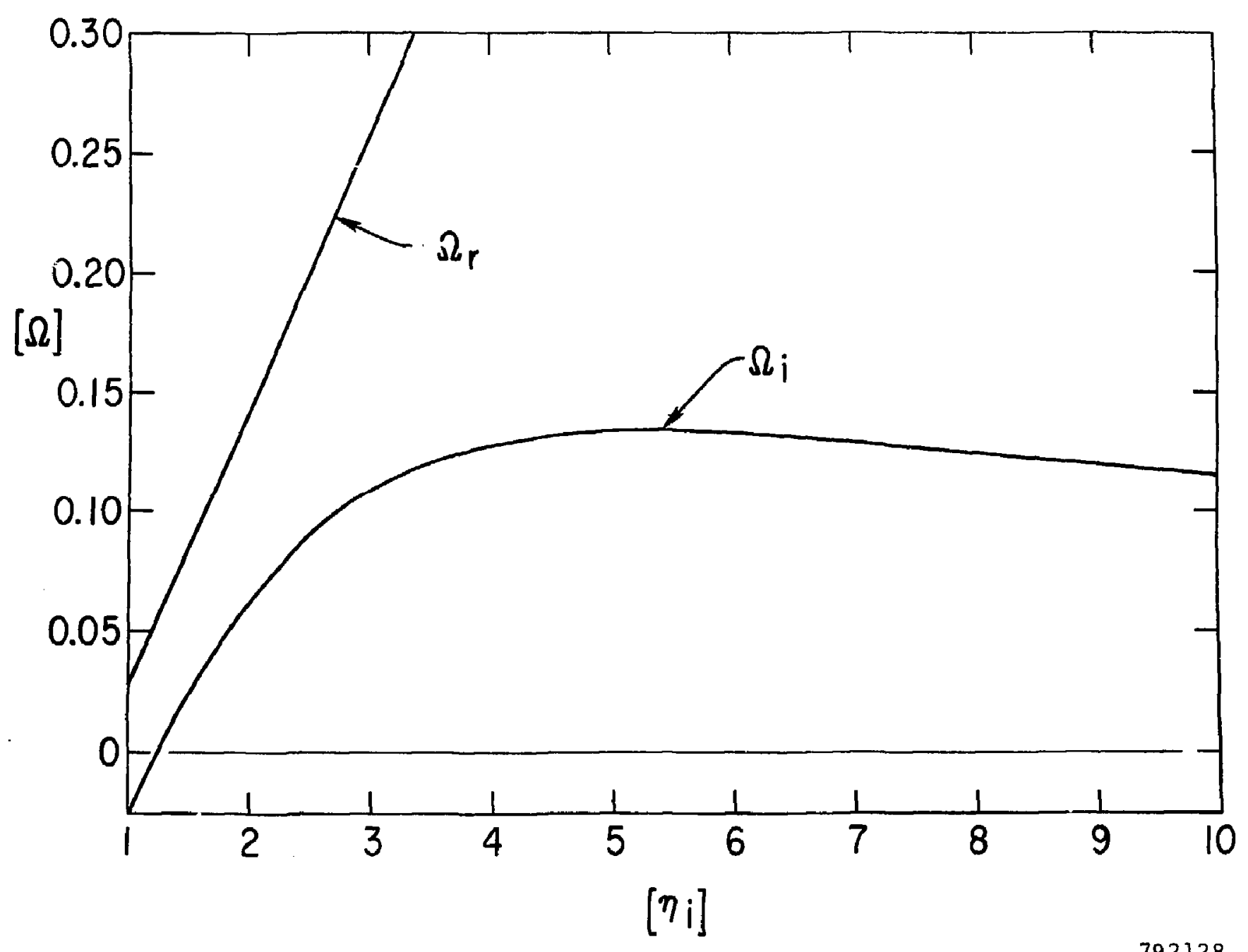

792128 Fig. 8. Ion temperature gradient dependence of $n_{i}$-driven instability with $\mathrm{b}=0.5, \mathrm{~L}_{\mathrm{n}} / \mathrm{L}_{\mathrm{s}}=0.1$, and $\mathrm{n}=0$. 


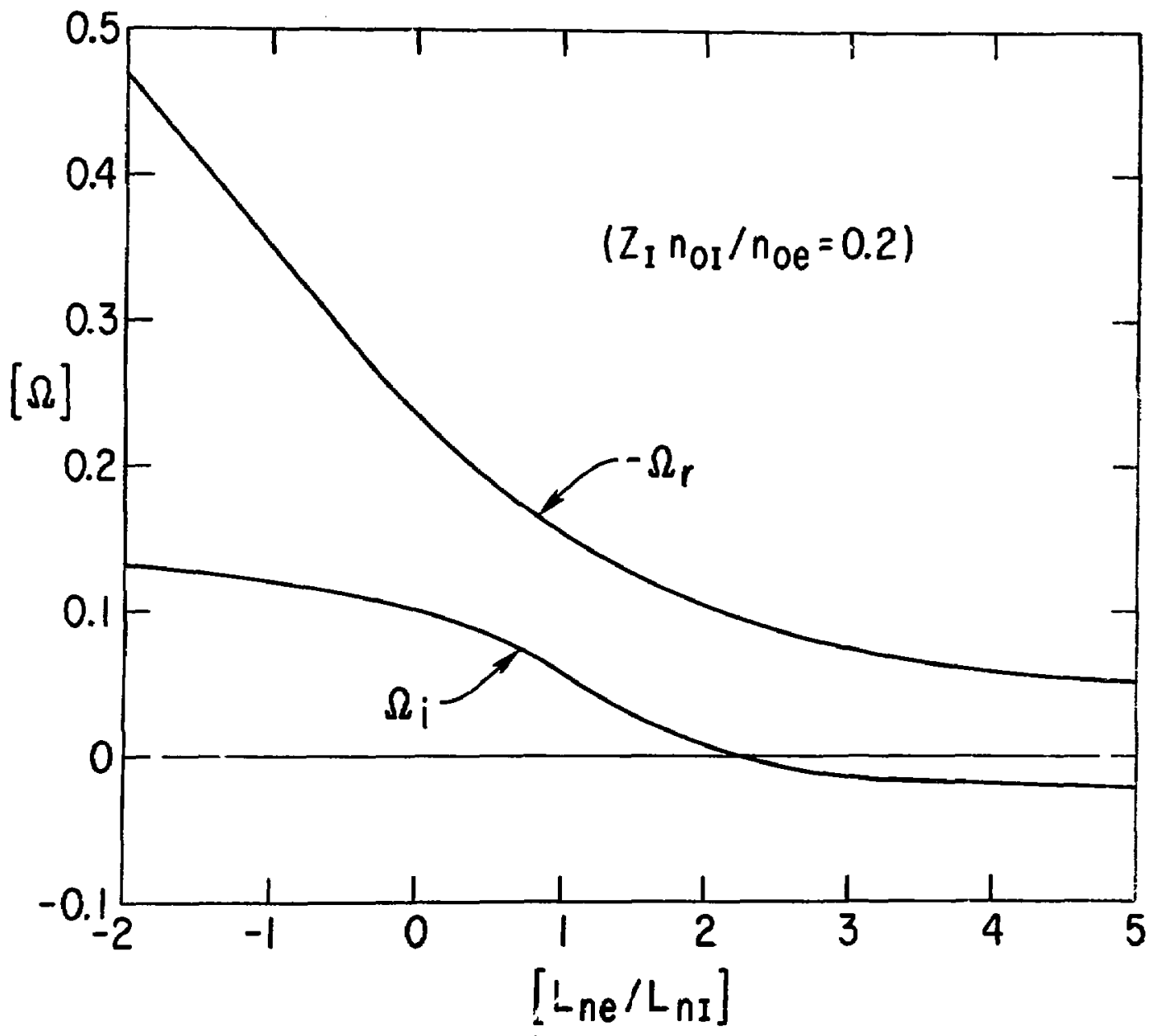

792122

Fig. 9. Typical stabilizing influence of impurities on $\eta_{i}$-driven instability as a function of degree of inward peaking of the impurity density profile, $\mathrm{L}_{\mathrm{ne}} / \mathrm{L}_{\mathrm{nI}}$. 


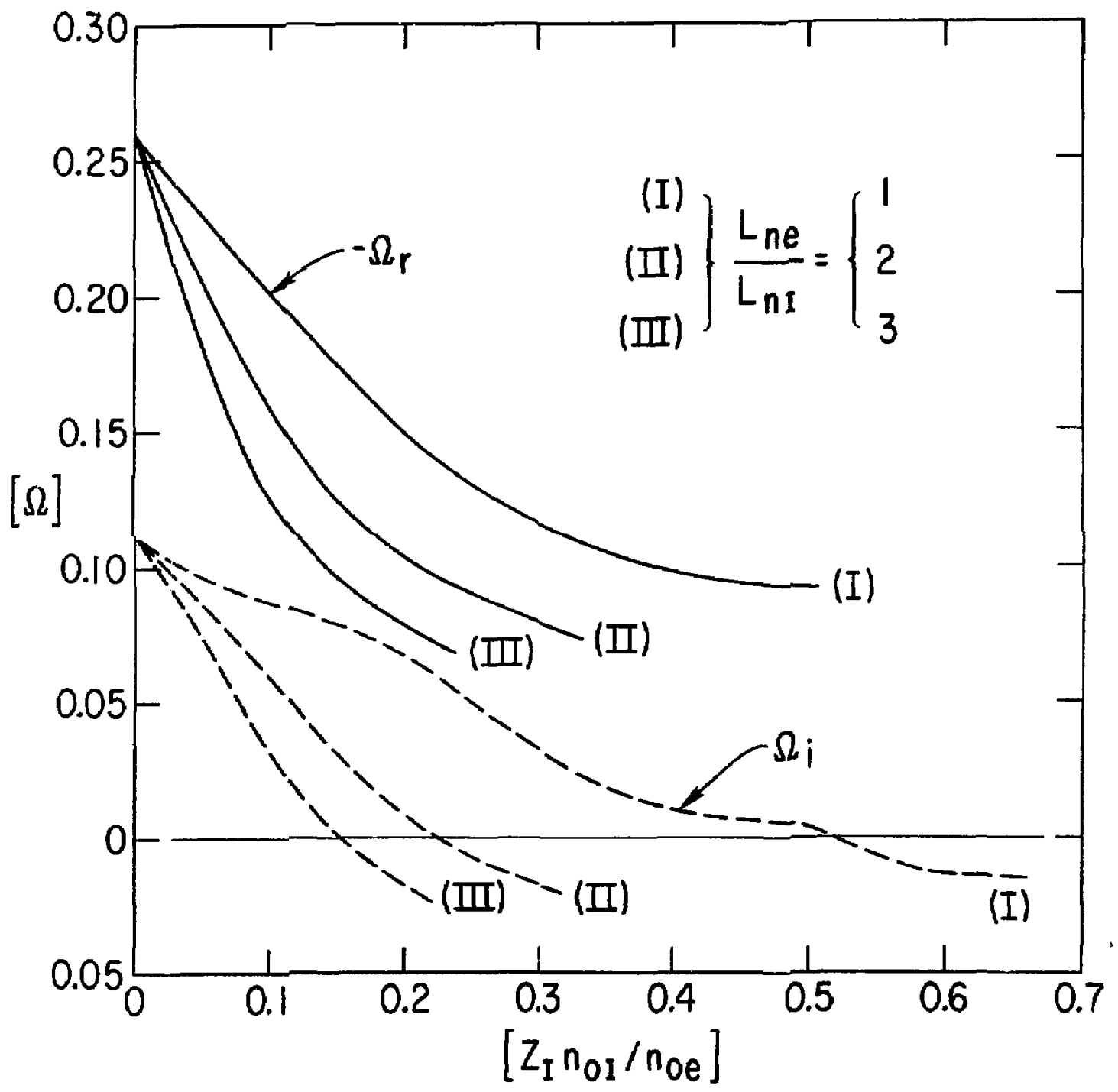

792125

Fig. 10. Typical stabilizing influence cf impurities on $\eta_{i}$-driven instability as a function of impurity concentration, $\mathrm{z}_{\mathrm{I}} \mathrm{n}_{\mathrm{oI}} / \mathrm{n}_{\mathrm{oe}}$, for a given degree of inward peaking of the impurity density profile, $\mathrm{L}_{\mathrm{ne}} / \mathrm{L}_{\mathrm{nI}}$. 